\title{
Ehrverletzung oder Gesellschaftskritik?
}

Von Tucholskys «Soldaten sind Mörder» zum päpstlichen Vergleich Abtreibung «ist so, als würde man einen Auftragsmörder mieten»

Nadine Zurkinden/Denise Buser *

Der drastische Vergleich des Papstes von Abtreibung und Auftragsmord wurde in der in- und ausländischen Presse aufgegriffen und vielfach kritisiert. Auch wenn die «Tathandlung» nicht in der Schweiz erfolgte, soll hier der Frage einer strafrechtlichen Ehrverletzung nachgegangen werden. Stand bei der Aussage die Kritik am gesellschaftlichen Umfeld, das schwangere Frauen zu wenig unterstützt, im Vordergrund? Kann man die Frauen als Kollektiv beleidigen?

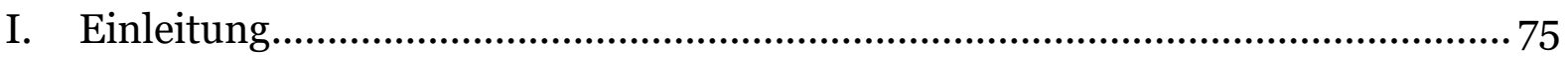

II. Meinungsäusserungsfreiheit und ihre Grenzen....................................................76

III. Diffamierung von Frauen als Verbrecherinnen: eine Ehrverletzung? ...................79

1. Vorbemerkung .............................................................................................. 79

2. Meinungsfreiheit vs. Schutz der Ehre ............................................................. 81

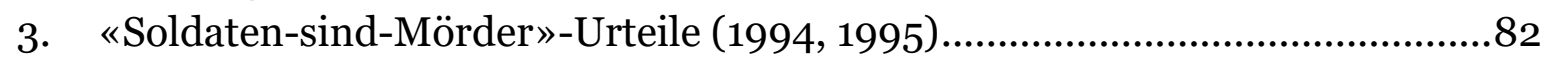

4. Anwendung der Kriterien auf den Papst-Vergleich.............................................84

IV. Sollte Geschlechterdiskriminierung strafbar sein?............................................... 88

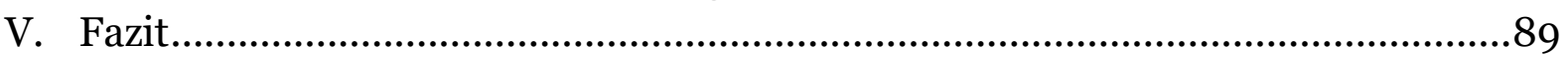

Zitiervorschlag: Nadine Zurkinden/Denise Buser, Ehrverletzung oder Gesellschaftskritik?, in: sui-generis 2019, S. 74

URL: $\quad$ sui-generis.ch/94

DOI: $\quad$ https://doi.org/10.21257/sg.94

\footnotetext{
Dr. iur. Nadine Zurkinden, Oberassistentin für Strafrecht und Strafprozessrecht, Rechtswissenschaftliches Institut der Universität Zürich, Präsidentin der Forschungsgemeinschaft Mensch im Recht. Prof. Dr. iur. Denise Buser, Titularprofessorin für kantonales öffentliches Recht, Juristische Fakultät der Universität Basel. Die Autorinnen danken Prof. Dr. iur. Patricia M. Schiess Rütimann (Titularprofessorin für öffentliches Recht und Verfassungsvergleichung an der Universität Zürich. Forschungsleiterin Recht am Liechtenstein-Institut in Bendern FL) für die kritische Durchsicht und wertvollen Bemerkungen zum Manuskript.
} 


\section{Einleitung}

1 In einer Generalaudienz ${ }^{1}$ hat Papst Franziskus am 10. Oktober 2018 auf dem Petersplatz zum fünften Gebot «Du sollst nicht töten» eine teilweise vom Blatt, teilweise frei gesprochene Rede gehalten. Darin thematisierte er auch den Schwangerschaftsabbruch, den er mit Auftragsmord verglich. Wörtlich sagte der Papst²:

«Un approccio contraddittorio consente anche la soppressione della vita umana nel grembo materno, in nome della salvaguardia di altri diritti. Ma come può essere terapeutico, civile o semplicemente umano un atto che sopprime la vita innocente e inerme nel suo sbocciare? Io vi domando: È giusto fare fuori una vita umana per risolvere un problema? Cosa pensate voi, è giusto? E giusto o no? [Gut vernehmbarer Zuruf aus dem Publikum: No.] È giusto affittare un sicario per risolvere un problema? [Gut vernehmbarer Zuruf aus dem Publikum: No.] Non si può, non è giusto fare fuori un essere umano - benché piccolo - per risolvere un problema. È come affittare un sicario per risolvere un problema.»

3 In der deutschen Übersetzung lautet die Passage wie folgt:

4 «Eine widersprüchliche Ansicht erlaubt auch die Unterdrückung des menschlichen Lebens im Mutterleib, unter dem Titel der Wahrung anderer Rechte. Aber kann eine Handlung, die unschuldiges

1 Die Generalaudienzen des Papstes finden in der Regel jeden Mittwoch um 10 Uhr auf dem Petersplatz vor dem Petersdom in Rom statt. Einlass bei der Audienz ist mit einer kostenlosen Eintrittskarte möglich, die man online bestellen kann.

2 Die Generalaudienz vom 10. Oktober 2018 ist auf der Homepage des Vatikans dokumentiert. Die entsprechende Passage erfolgt ca. bei Minute 13. und wehrloses Leben bei seinem Aufblühen vernichtet, als therapeutisch, zivil oder gar menschlich bezeichnet werden? Ich frage Euch: Ist es richtig, ein menschliches Leben aus der Welt zu schaffen, um ein Problem zu lösen? Was denkt Ihr, ist es richtig? Ist es richtig, ja oder nein? [Gut vernehmbarer Zuruf aus dem Publikum: Nein.] Ist es richtig, einen Auftragsmörder zu engagieren, um ein Problem zu lösen? [Gut vernehmbarer Zuruf aus dem Publikum: Nein.] Das geht nicht, es ist nicht richtig, ein Menschenwesen - und sei es noch so winzig - aus der Welt zu schaffen, um ein Problem zu lösen. Es ist so, als würde man einen Auftragsmörder engagieren, um ein Problem zu lösen.»3

5 Auch wenn der Papst auf Italienisch spricht, sind seine Worte aus seiner Sicht wohl an alle Menschen, mindestens aber an alle Katholiken und Katholikinnen der Welt gerichtet. Betroffen sind vom letzten Satz allerdings primär Frauen, da sie pro futuro nicht wissen, ob sie jemals in die inkriminierte Situation kommen. 4

6 Es ist festzuhalten, dass sich auch Männer durch den Papst-Vergleich (Abtreibung sei so, als würde man einen Auftragsmörder engagieren) beleidigt fühlen können, sei es als Väter, die ihren Töchtern helfen wollen, als Ehemänner, die

\footnotetext{
Übersetzung durch die Autorinnen.

Nicht alle betroffenen Frauen müssen sich persönlich beleidigt fühlen. Es können aber auch Frauen betroffen sein, an die man nicht zuallererst denkt, wie etwa Nonnen. Siehe dazu den Dokumentarfilm von Eric Quintin und Marie-Pierre Raimbault, Gottes missbrauchte Dienerinnen, Frankreich 2017. Verfügbar bis zum 16.04.2019. Ebenso spricht der Vergleich des Papstes ein Unwerturteil über Frauen aus, die zwar nie abgetrieben haben, aber - unter Umständen - dazu bereit gewesen wären oder einen Schwangerschaftsabbruch zumindest nicht jederzeit kategorisch abgelehnt haben.
} 
den Entscheid ihrer Frau unterstützen, als verständnisvolle Arbeitskollegen, als Angestellte einer medizinischen Einrichtung etc. Allerdings sind sie insofern weniger betroffen, als Schwangerschaft und Abtreibung sie nicht körperlich betreffen. Männer können weder (z.B. ungewollt durch Vergewaltigung) schwanger werden, noch müssen sie den Entscheid über ihren Körper treffen und die Einwilligung zum Eingriff geben, sondern (hoffentlich!) die Frau. Der päpstliche Vergleich ist zudem pauschal und differenziert nicht zwischen strafbaren und straflosen Abbrüchen. 5 Letztere meint er sogar explizit mit, wenn er sagt: «Un approccio contraddittorio consente anche la soppressione della vita umana nel grembo materno, in nome della salvaguardia di altri diritti.»

7 Der drastische Vergleich wurde in der inund ausländischen Presse aufgegriffen und verbreitet. Vielfach wurde er auch unter Empörung zurückgewiesen. ${ }^{6}$ Auch wenn die «Tathandlung» in Vatikanstadt7 in Rom erfolgte, soll hier untersucht werden, ob es sich bei diesen Aussagen um eine strafbare Ehrverlet-

5 Siehe für die Schweiz Art. 118 und 119 StGB Schweizerisches Strafgesetzbuch (SR 311.0).

6 Siehe etwa IG Feministische Theologinnen Deutschschweiz/Liechtenstein. Siehe auch die Petition «Abtreibung ist kein Verbrechen» des Diözesanverbandes Berlin des Deutschen Katholischen Frauenbundes.

7 Die Vatikanstadt bzw. der Vatikan ist ein Völkerrechtssubjekt (aber seine Staatlichkeit ist fraglich). Der Heilige Stuhl mit dem Papst als Inhaber ist ebenfalls Völkerrechtssubjekt und vom Vatikan zu unterscheiden. Beide können jedoch grundsätzlich völkerrechtliche Verträge unterzeichnen; Matthias Herdegen, Völkerrecht, 17. Aufl., München 2018, S. 97 Rn. 1; Volker Epping/Wolff Heintschel von Heinegg (Hrsg.), Völkerrecht, Ein Studienbuch, 7. Aufl., München 2018, S. 437 Rn. 1 ff.; Anne Peters, Völkerrecht, Allgemeiner Teil, 4. Aufl. Zürich/Basel/Genf 2016, Kap. 10 Rn. 5 ff. zung nach schweizerischem Recht handeln könnte. Immerhin wurde die Aussage des Papstes auch von offiziellen katholischen Medien in der Schweiz weiterverbreitet. ${ }^{8}$ Dabei wird auch ein vergleichender Blick auf die «Soldaten-sindMörder»-Urteile des deutschen Bundesverfassungsgerichts von 1994 und 1995 geworfen. Die dort im Zentrum stehenden Fragen, ob eine Äusserung an sich diffamierend oder «bloss» gesellschaftskritisch und damit von der Meinungsäusserungsfreiheit gedeckt ist, und ob Gruppen ehrverletzungsfähig sind, stellen sich auch bei einer strafrechtlichen Würdigung des päpstlichen Vergleichs.

\section{Meinungsäusserungsfreiheit und ihre Grenzen}

8 Das Grundrecht der Meinungsäusserungsfreiheit wird in Art. 16 der Bundesverfassung ausdrücklich garantiert, nachdem es zuvor seit 1965 vom Bundesgericht als ungeschriebenes Freiheitsrecht anerkannt worden war. 9 Im internationalen Recht wird es in Art. 10 EMRK ${ }^{10}$ und Art. 19 UNO-Pakt II11 gewährleistet. Die Meinungsäusserungsfreiheit wird mit einer Reihe anderer Grundrechte, wie etwa die Medienfreiheit, die Kunstfreiheit und die Versammlungsfreiheit, als Teil der Grundrechte freier Kommunikation ver-

$8 \overline{\text { Siehe z.B. «Papst vergleicht Abtreibung mit Auf- }}$ tragsmord» vom katholischen Medienzentrum.

9 Siehe dazu: Jörg Paul Müller/Markus Schefer, Grundrechte in der Schweiz, Im Rahmen der Bundesverfassung, der EMRK und der UNOPakte, 4. Aufl., Bern 2008, S. 437.

10 Die europäische Menschenrechtskonvention (SR o.101) ist für die Schweiz 1974 in Kraft getreten.

11 Der internationale Pakt über bürgerliche und politische Rechte (SR 0.103.2) ist für die Schweiz 1992 in Kraft getreten. 
standen. ${ }^{12}$ Wegen der elementaren Bedeutung der freien Kommunikation in einer demokratischen Gesellschaft stehen die Meinungsäusserungsfreiheit und die anderen Kommunikationsgrundrechte «unter besonderem verfassungsrechtlichen Schutz.»13 Bereits in einem Urteil von 1976 hielt der Europäische Gerichtshof für Menschenrechte fest: «Das Recht der freien Meinungsäusserung (...) gilt (...) nicht nur für die günstig aufgenommenen oder als unschädlich oder unwichtig angesehenen Informationen, oder Gedanken, sondern auch für die, welche den Staat oder irgendeinen Bevölkerungsteil verletzen, schockieren oder beunruhigen. So wollen es Pluralismus, Toleranz und Aufgeschlossenheit, ohne die es eine demokratische Gesellschaft nicht gibt.» ${ }^{14}$ Hier kommt zum Ausdruck, dass die Kommunikationsfreiheiten geradezu im öffentlichen Interesse einer freiheitlichen Gesellschaft liegen.15 Deshalb sind «Einschränkungen von Äusserungen, die an den Inhalt der Kommunikation anknüpfen (...) an einem strengen Massstab zu messen.»16 Müller/Schefer

12 Müller/Schefer (Fn. 9), S. 347 ff.; Giovanni Biaggini, BV Kommentar, 2. Aufl., Zürich 2017, Art. 16 Rz. 3; zum Begriff auch BGE 140 III 616, 630.

13 Müller/Schefer (Fn. 9), S. 348.

14 Urteil des EGMR 5493/72 vom 7.12.1976 (Handyside v. Vereinigtes Königreich), § 49; deutsche Übersetzung aus EuGRZ 1977, S. 38 ff., 42 (Übersetzung).

15 Dies hat der Europäische Gerichtshof für Menschenrechte seither immer wieder betont. Siehe z.B. Urteil des EGMR 20261/12 vom 23.6.2016 (Baka v. Ungarn), § 158.

16 Müller/Schefer (Fn. 9), S. 383. Man kann sich noch die Frage stellen, in welchem Verhältnis die Meinungsfreiheit (Art. 16 BV) und das Diskriminierungsverbot (Art. 8 Abs. 2 und 3 BV) stehen. Beides sind gleichrangige Grundrechte, d.h. man darf beispielsweise die Meinung vertreten, dass das Diskriminierungsverbot falsch sei. Erst wenn sich die Art und Weise einer solchen Meinungsäusserung zu einer Ehrverletzung verdichtet, kommt man wieder an die Grenze der Meinungsfreiheit. weisen darauf hin, dass sonst die Gefahr bestehe, «dass mit den strafrechtlichen Ehrverletzungstatbeständen weniger das Ansehen und die Würde» der angegriffenen Personen geschützt, "sondern missliebige Kritiker zum Schweigen gebracht werden sollen.»17

9 Gleichwohl ist im schweizerischen und internationalen Recht anerkannt, dass es unter klar formulierten Voraussetzungen auch Grenzen für die Meinungsäusserungsfreiheit gibt. So verfügen Kommunikationsinhalte über einen entsprechend geringeren Grundrechtsschutz, wenn sie rassistische, Religionsüberzeugungen verletzende, pornografische und ehrverletzende Elemente aufweisen. Es gibt ein öffentliches Interesse an der Verhinderung oder Einschränkung solcher Äusserungen, weshalb sie unter Beachtung des Verhältnismässigkeitsprinzips entsprechend unterbunden werden können. ${ }^{18}$ Illustrativ ist etwa das Urteil des deutschen Bundesverfassungsgerichts aus dem Jahr 2006, wo Kritiker von Abtreibungskliniken einen Arzt als «Tötungs-Spezialist[en] für ungeborene Kinder» bezeichneten. Eine solche Äusserung ist durch die Meinungsfreiheit geschützt. Nicht geschützt wurde jedoch die Bezeichnung der Arbeit desselben Arztes als «Babycaust» unter

17 Müller/Schefer (Fn. 9), S. 391.

18 Müller/Schefer (Fn. 9), S. 383 ff. mit ausführlichen Angaben zur EGMR-Rechtsprechung. Siehe in neuerer Zeit z.B. Urteil des EGMR 18597/13 vom 9.1.2018 (GRA Stiftung gegen Rassismus und Antisemitismus v. Switzerland); der Europäische Gerichtshof für Menschenrechte hob darin ein Urteil des Bundesgerichts auf, das es einer Stiftung verboten hatte, die Aussagen eines SVPPolitikers im Zusammenhang mit der MinarettInitative als «verbalen Rassismus» zu bezeichnen. Der Gerichtshof in Strassburg betonte die mit der Presse vergleichbare Rolle der Stiftung als «public watchdog». 
Bezugnahme auf den Holocaust. ${ }^{19}$ Das Bundesverfassungsgericht hatte diese Äusserung als Angriff auf die Menschenwürde des betroffenen Arztes qualifiziert. In der Begründung hielt es fest, dass bei wertenden Äusserungen der Persönlichkeitsschutz gegenüber der Meinungsfreiheit zurücktrete, es sei denn die Äusserung ziele ausschliesslich auf Beleidigung ab oder stelle einen Angriff auf die Menschenwürde des Adressaten dar. ${ }^{20}$ In der Schweizer Rechtsprechung wurde etwa

$19 \overline{\text { Urteil des Bundesverfassungsgerichts } 1 \text { BvR 49/0O }}$ (2006). Im gleichen Verfahren wurde ein zivilrechtlicher Unterlassungsanspruch in Bezug auf die Formulierung «Kindermord im Mutterschoss» bejaht (Urteil des BVerfG, 1 BvR 49/oo, Rn. 66, 67).

20 Urteil des BVerfG, 1 BvR 49/oo (Fn. 19), Rn. 57. Der Europäische Gerichtshof für Menschenrechte schützte diesen Entscheid und sah die Meinungsäusserungsfreiheit einstimmig nicht als verletzt an (Urteil des EGMR 397/07 und 2322/07 vom 13.1.2011 [Hoffer und Annen v. Deutschland]). Auch in den meisten weiteren Urteilen betreffend denselben Beschwerdeführer und ähnliche Fälle (meist betreffend zivilrechtliche Urteile) schützte der EMRK die Urteile der deutschen Gerichte und sah jeweils einstimmig keine Verletzung der Meinungsäusserungsfreiheit (Urteil des EGMR 3682/10 vom 20.9.2018 [Annen v. Germany Nr. 2]; Urteil des EGMR 3687/10 vom 20.9.2018 [Annen v. Germany Nr. 3]; Urteil des EGMR 9765/10 vom 20.9.2018 [Annen v. Germany $\mathrm{Nr}$ 4]; Urteil des EGMR 70693/11 vom 20.9.2018 [Annen v. Germany Nr. 5]; Urteil des EGMR 3779/11 vom 18.10.2018 [Annen v. Germany Nr. 6]). Nur im Urteil des EGMR 3690/10, vom 26.11.2016 (Annen v. Deutschland), hat Annen betreffend eines anderen Flyers Recht bekommen, weil fünf von sieben Richtern unter anderem auf eine zweite Referenzebene im Flyer verwiesen: «In fact, the applicant's statement according to which the killing of human beings in Auschwitz had been unlawful, but allowed, and had not been subject to criminal liability under the Nazi regime, may also be understood as a way of creating awareness of the more general fact that law may diverge from morality» (Rn. 63). Zwei Richterinnen widersprachen in einer joint dissenting opinion diesem Urteil, da Annen mit dem Flyer nicht nur an einer moralischen und ethischen Debatten teilgenommen habe, sondern explizit zwei Ärzte exponierte, um ihrem Ruf zu schaden. zugunsten des strafrechtlichen Ehrverletzungsschutzes entschieden, wenn beim Pressebericht über einen noch nicht rechtskräftig verurteilten Straftäter zusätzlich die Unschuldsvermutung zu beachten war (BGE 116 IV 31), oder wenn bei einem Presseerzeugnis die Kombination von verschiedenen Elementen der grafischen Darstellung und des textlichen Inhalts in seiner Gesamtheit ehrverletzend war. ${ }^{21}$

10 Besonders geschützt werden durch das Bundesgericht ${ }^{22}$ und den Europäischen Gerichtshof für Menschenrechte ${ }^{23}$ auch kritische bzw. verletzende Äusserungen, wenn sie sich an öffentliche Personen aus Politik und Wirtschaft richten. Gewählte Politiker und Politikerinnen müssen sich intensivste Kritik, auch verletzende, gefallen lassen. ${ }^{24}$ Wenn die angegriffene

21 BGE 131 IV 160 = Pra 95 (2006) Nr. 59; BGE 137 IV $313=$ Pra 101 (2012) Nr. 53.

22 Siehe dazu Müller/Schefer (Fn. 9), S. 391 f. mit zahlreichen Rechtsprechungsangaben. Ein illustratives Beispiel für satirische Ehrverletzung stellt das Urteil des Bundesgerichts 5A_553/2012 vom 14. April 2014 dar, in dem es um Nacktdarstellungen in Bildmontagen von bekannten Managern wie etwa Daniel Vasella ging. Ein weiteres Beispiel, das grosse mediale Beachtung fand, ist die «Schmähkritik» über den türkischen Staatspräsidenten, die im Rahmen einer Fernsehsendung in Deutschland vorgetragen wurde. Siehe dazu Klaus HoffmannHolland/Johannes Koranyi, ZStW 2018, S. 82-105. Die Autoren plädieren für einen strafrechtsfreien Raum für die Satire. Betroffene sollen vielmehr einen in die Zukunft gerichteten zivilrechtlichen Unterlassungsanspruch geltend machen. Freilich bleibt damit dennoch ein Damoklesschwert über der Satire hängen: Wenn beispielsweise ein vorläufiger Unterlassungsanspruch erfolgreich ist, kann dies den Abbruch einer ganzer Theatersaison bewirken, was den Künstler einem doppelten Kostenrisiko aussetzt.

23 Siehe Urteil des EGMR 20261/12 vom 23.6.2016 (Baka v. Ungarn), §§ 159, 168, 170 f. (hier ging es um die Kritik eines entlassenen Gerichtspräsidenten an der Gerichtsreform der regierenden FideszPartei in Ungarn).

24 Siehe Urteil des Bundesgerichts 6B_1270/2017 und 6B_1291/2017 vom 24. April 2018, E. 2.4.2, 2.4.3. 
Person zwar nicht Inhaberin eines politischen Amts ist, aber doch eine gewisse Aufmerksamkeit in der Öffentlichkeit auf sich zieht, darf sie «ebenfalls intensiver kritisiert werden als Privatpersonen».25

\section{Diffamierung von Frauen als Ver- brecherinnen: eine Ehrverletzung?}

\section{Vorbemerkung}

11 Der Fokus dieses Beitrags liegt auf der Frage, ob die päpstliche Äusserung eine strafrechtliche Ehrverletzung darstellt oder ob sie von der Meinungsfreiheit gedeckt ist. Auf die Frage nach Jurisdiktion und Immunität und auf die Qualifizierung der päpstlichen Aussage als Werturteil oder Tatsachenbehauptung wird deswegen nur im Rahmen dieser Vorbemerkungen kurz eingegangen.

\section{a) Jurisdiktion}

Das Schweizer Strafrecht ist auf den Papst und seine Aussage in der Schweiz aus zwei Gründen nicht anwendbar:

Erstens geniesst der Papst grundsätzlich Immunität vor nationalen Gerichten. ${ }^{26}$
25 Müller/Schefer (Fn. 9), S. 392. Müller/Schefer führen als Beispiele Förster, Gefängniswärter, Polizisten oder Offiziere der Armee an.

26 Joanne Foakes, The Position of Heads of State and Senior Officials in International Law, Oxford 2014, S. 30. In den USA hatte aber ein Bundesgericht entschieden, in einem Missbrauchsskandal die Immunität des Heiligen Stuhls aufzuheben. Dieser Entscheid wurde 2010 vom obersten Gerichtshof in Washington gestützt Der internationale Strafgerichtshof (IStGH) hat sich mit der Immunitäts-Frage nicht auseinandergesetzt: Laut dessen Ankläger unterlag eine von SNAP (Survivors Network of those abused by priests) im Sep-
14 Zweitens zeitigt seine Aussage wohl keinen Erfolg im Sinne von Art. 8 Abs. 1 StGB in der Schweiz, ${ }^{27}$ was Voraussetzung für die Anwendbarkeit des Schweizer Strafrechts wäre. Sinnvollerweise ist dem Bundesgericht folgend ein Erfolg im Sinne von Art. 8 StGB nur anzunehmen, wenn (ehrverletzende) Äusserungen aus dem Ausland an individuell bestimmte Personen in der Schweiz gerichtet und von diesen zur Kenntnis genommen werden. ${ }^{28}$

$15 \mathrm{Ob}$ auch bei ehrverletzenden Äusserungen, die im Ausland ins Internet gestellt und nicht zielgerichtet an eine individuell bestimmte Person in der Schweiz gerichtet, aber in der Schweiz zur Kenntnis genommen werden, ein Erfolg i.S.v. Art. 8 StGB vorliegt, ist unklar. ${ }^{29}$ Wird er be-

tember 2011 eingereichte Klage gegen den damaligen Papst Benedikt nicht der Gerichtsbarkeit des Strafgerichtshofs. Siehe dazu die Pressemittelung von SNAP. Zur Frage der Beachtlichkeit völkerrechtlicher Immunitäten für den IStGH siehe Helmut Kreicker, Immunität und IStGH, ZIS 2009, S. 350-367.

27 Als Erfolg gilt eine Veränderung in der Aussenwelt: BGE 105 IV 326; Sabine Gless, Internationales Strafrecht, 2. Aufl., Basel 2015, Rn. 156. Ob Erfolg im Sinne von Art. 8 StGB und Erfolg im Sinne der Dogmatik der Deliktscharakterisierung gleich sind, ist eine andere Frage. Dazu Gless (Fn. 27), Rn. 159.

28 BGE 125 IV 177, E. 2 und 3. Hier ging es um ehrverletzende Äusserungen in einem Brief aus dem Ausland, der zielgerichtet an individuell bestimmte Personen in der Schweiz versandt und von den Adressaten in der Schweiz zur Kenntnis genommen wurden.

29 Franz Riklin, in: Basler Kommentar, Strafgesetzbuch II, 4. Aufl., Basel 2018, vor Art. 173 N 50a; Stefan Trechsel/Viktor Lieber, in: Schweizerisches Strafgesetzbuch. Praxiskommentar, 3. Aufl., Zürich 2017, vor Art. 173 N 12a; Gless (Fn. 27), Rn. 157 ff., Rn. 162; Christian Schwarzenegger, Der räumliche Geltungsbereich des Strafrechts im Internet. Die Verfolgung von grenzüberschreitender Internetkriminalität in der Schweiz im Vergleich mit Deutschland und Österreich, ZStrR 118 (2000), S. 124; Jann C. Six, Die Beschimpfung im Internet, in: Jürg-Beat Ackermann (Hrsg.), Strafrecht als Herausforderung, Zürich 1999, S. $328 \mathrm{ff}$. 
jaht, würde die Schweiz zum virtuellen Weltpolizisten, und wer im Ausland Inhalte ins Netz stellt, müsste vorher Rechtsauskünfte in der Schweiz (und jedem anderen Land, das den räumlichen Geltungsbereich seines Strafrechts extensiv auslegt) einholen, selbst wenn die Inhalte nach ausländischem Recht legal wären. ${ }^{30}$ Auch ein Abstellen auf die Wahrscheinlichkeit der Kenntnisnahme aufgrund konkreter Umstände, wie Sprache und Ausrichtung der Äusserung, erscheint uns zu weit, ${ }^{31}$ zumal der Papst in der übers Web übertragenen Generalaudienz zwar italienisch spricht, sie aber weltweit auch von nicht italienisch sprechenden Menschen zur Kenntnis genommen wird.

Dennoch ist es interessant, die Strafbarkeit der Äusserung in der Schweiz zu prüfen. Denn sie könnte auch von einem Schweizer Bischof wiederholt werden, der sich die Äusserung zu eigen macht.

\section{b) Werturteil oder Tatsachenbe- hauptung?}

17 Welcher Ehrverletzungstatbestand betroffen sein könnte, ergibt sich aus der Qualifikation der Aussage, die der Papst gleichzeitig allfälligen Betroffenen und Dritten gegenüber macht. Man unterscheidet zwischen Tatsachenbehauptungen und Werturteilen. Tatsachenbehauptungen gegenüber Dritten können den Tatbestand der üblen Nachrede (Art. 173 StGB) oder der Verleumdung (Art. 174 StGB) erfüllen. Werturteile gegenüber Dritten können den Tatbestand der Beschimpfung (Art. 177 StGB) erfüllen. Bei den Äusserungen des Papstes ist fraglich, welche Variante vorliegt.

$30 \quad$ Six (Fn. 29), S. 329.

31 So aber etwa Six (Fn. 29), S. 330 f.
18 Tatsachenbehauptungen sind gemäss BGE 118 IV 41 m.w.Nw. «Ereignisse oder Zustände der Gegenwart oder Vergangenheit [...], die äusserlich in Erscheinung treten und dadurch wahrnehmbar und dem Beweise zugänglich werden.»

19 Eine Tatsachenbehauptung wäre etwa die Aussage: «Frau X hat einen Auftragsmörder engagiert.» Es wäre eine Tatsache, die sich überprüfen liesse. Bei der Aussage des Papstes, Abtreibung sei so, als würde man einen Auftragsmörder anheuern, meint der Papst generell, dass ein Schwangerschaftsabbruch dasselbe ist, wie einen Auftragsmörder zu engagieren. Er bezieht sich dabei nicht nur auf vergangene oder gerade erfolgende Schwangerschaftsabbrüche, sondern auch auf künftige. Damit scheidet eine Tatsachenbehauptung aus. Der Papst gibt eine Wertung zum Schwangerschaftsabbruch $a b$, indem er diesen mit einem Auftragsmord vergleicht. $3^{2}$ Die Unterscheidung zwischen Tatsachenbehauptungen und

$32 \overline{\text { Dieser Vergleich ist anders gelagert als der Sach- }}$ verhalt, der BGE 128 IV 53 zugrunde lag. Das Bundesgericht ging dort aufgrund der Gesamtumstände von einer Tatsachenbehauptung aus (nicht von einem Werturteil): Die Beschuldigten hatten über bestimmte Befürworterinnen einer Fristenregelung auf einem Plakat geschrieben, «Elles veulent une culture de la mort en Suisse!» und unter dieser Aussage einen blutigen etwa 20 Wochen alten Fötus abgebildet mit der Bildunterschrift «L'empoisonner, le découper à la curette ou le laisser mourir dans une poubelle?». Das Bundesgericht äusserte sich dazu folgendermassen: «L'accusation de vouloir une culture de la mort, à l'appui de la photo figurant sur l'affiche, revient à alléguer que les intimées souscrivent au découpage, à l'empoisonnement ou à l'abandon dans une poubelle de foetus bien développés. Par ailleurs, l'expression 'culture de la mort' ne contient pas d'invective et n'est pas non plus un terme grossier dont il conviendrait de déterminer s'il est propre à attaquer la victime dans son honneur. L'expression litigieuse constitue par conséquent une allégation de fait et non un jugement de valeur.» (BGE 128 IV 53, E. 1.f/bb). 
Werturteilen ist auch für die Beurteilung der Grenzen der Meinungsäusserungsfreiheit relevant. Dabei qualifiziert der Europäische Gerichtshof für Menschenrechte Vergleiche regelmässig als Werturteile. 33 Auch das Deutsche Bundesverfassungsgericht qualifiziert im Babycaust-Urteil die Aussage «Kinder-Mord im Mutterschoss» als «ein das Persönlichkeitsrecht beeinträchtigendes Werturteil».34 Ebenso ging das Deutsche Bundesverfassungsgericht im «Soldaten-sind-Mörder»-Urteil35 von 1995 von einem Werturteil aus. Es führte aus: «Meinungen sind im Unterschied zu Tatsachenbehauptungen durch die subjektive Einstellung des sich Äußernden zum Gegenstand der Äußerung gekennzeichnet [...]. Sie enthalten sein Urteil über Sachverhalte, Ideen oder Personen.» 36 «Die Beschwerdeführer haben mit ihren Äußerungen, Soldaten seien Mörder oder potentielle Mörder, nicht von bestimmten Soldaten behauptet, diese hätten in der Vergangenheit einen Mord begangen. Sie haben vielmehr ein Urteil über Soldaten und über den Soldatenberuf zum Ausdruck gebracht, der unter Umständen zum Töten anderer Menschen zwingt.» 37

33 Siehe dazu Florian Zihler, Tatsachenaussagen und Werturteile: Eine überholte Kategorisierung?, in: Jusletter 15. August 2005, Rn. 14 und 16 mit Beispielen zur Rechtsprechung. Neuere Urteile, in denen diese Unterscheidung angesprochen wird, sind Urteil des EGMR 38450/12 vom 25.10.2018 (E.S. v. Austria); Urteil des EGMR 3779/11 vom 18.10.2018, (Annen v. Germany [Nr. 6, siehe Fn. 20]); Urteil des EGMR 27447/07 vom 8.10.2015 (Kharmalov v. Russia); Urteil des EGMR 26671/09 vom 22.1.2015 (Pinto Pinheiro Marques v. Portugal).

34 Urteil des BVerfG, 1 BvR 49/oo (Fn. 19), Rn. 63.

35 Siehe 3.c).

36 Urteil des BVerfG, 1 BvR 1476/91 (1995), Rn. 108 m.w.Nw.

37 Urteil des BVerfG, 1 BvR 1476/91 (Fn. 36), Rn. 109.
20 Ähnlich lässt sich auch bei der Aussage des Papstes argumentieren, dass er nicht von einer bestimmten Frau behauptet hat, dass sie einen Mord in Auftrag gegeben hätte, oder von einem bestimmten Arzt oder einer bestimmten Ärztin, dass er oder sie einen Auftragsmord ausgeführt hätte. Stattdessen bewertet der Papst den Schwangerschaftsabbruch als Mord. Seine Äusserung ist damit als Werturteil zu qualifizieren und als strafrechtlich relevanter Straftatbestand kommt Art. 177 StGB (Beschimpfung) in Frage.

\section{Meinungsfreiheit vs. Schutz der Ehre}

21 Ob das Werturteil strafbar ist, hängt $a b$ von einer Güterabwägung zwischen der verfassungsrechtlich geschützten Meinungsäusserungsfreiheit und dem strafrechtlichen Schutz der Ehre, der seinerseits Ausdruck von Grundrechten ist (Achtung der Menschenwürde, Art. 7 BV, und Schutz der Privatsphäre, Art. 13 BV). ${ }^{38}$

22 Laut Bundesgericht spielt es keine Rolle, ob aus einem Grundrecht (wie der Meinungsäusserungsfreiheit) ein Rechtfertigungsgrund hergeleitet wird, oder die gleichen Gesichtspunkte an einem anderen Ort einfliessen. 39

23 Zentral ist, dass die Güterabwägung jeweils anhand der gesamten konkreten Umstände vorzunehmen ist. Generelle Regeln sind schwierig aufzustellen. Um diese Güterabwägung für den PapstVergleich vorzunehmen, lohnt es sich, zunächst einen Blick über die Grenze auf die «Soldaten-sind-Mörder»-Urteile zu

38 BGE 131 IV 160 = Pra 95 (2006) Nr. 59, E. 3.3.1.

39 BGE 118 IV 153, E. 4.c. 
werfen. Denn auch die deutsche Praxis nimmt bei der Beurteilung von verletzenden Äusserungen eine Güterabwägung zwischen den Interessen an der Meinungsfreiheit und den Interessen am Schutz der persönlichen Ehre vor.

\section{3. «Soldaten-sind-Mörder»-Urteile (1994, 1995)}

\section{a) Sachverhalte}

241994 und 1995 hob das deutsche Bundesverfassungsgericht in den sogenannten «Soldaten-sind-Mörder»-Urteilen vorinstanzliche Strafurteile wegen Verletzung der Meinungsäusserungsfreiheit (Art. 5 Grundgesetz Deutschland) auf. Das deutsche Bundesverfassungsgericht hatte schon 1992 im gleichen Sinn entschieden, als eine Rubrik in der satirischen Zeitschrift Titanic im Zentrum stand, in der eine Einzelperson, die trotz Querschnittslähmung in den Wehrdienst wollte, als «geb. Mörder» bezeichnet wurde und im Begleittext die Bezeichnung eines Soldaten als «potentieller Mörder» erschien.40 Im Folgenden soll die Argumentation des letzten «Soldatensind-Mörder»-Urteils von 1995 (SoldatenMörder II) näher angeschaut werden, dem

$40 \overline{\text { Urteil des BVerfG, } 1 \text { BvR 514/90 (1992). Im Po- }}$ tentielle-Mörder-Urteil von 1992 lag dem deutschen Bundesverfassungsgericht folgender Sachverhalt vor: Die satirische Zeitschrift Titanic enthielt die ständige Rubrik «Die sieben peinlichsten Persönlichkeiten». Darin wurden jeweils meist öffentlich bekannte Personen mit einem schlagwortartigen Namenszusatz genannt. In der MärzAusgabe 1988 wurden drei Namen mit dem $\mathrm{Zu}-$ satz «geb.» ergänzt, darunter der damalige Bundespräsident als «Richard von Weizsäcker (geb. Bürger)» aufgeführt. Danach folgte der Kläger des Ausgangsverfahrens mit Vor- und Zunamen und mit dem Klammerzusatz «geb. Mörder». Bei diesem Kläger handelte es sich um einen querschnittsgelähmten jungen Mann, dem es gelang, in die Bundeswehr aufgenommen zu werden. vier unterschiedliche Sachverhalte zur Beurteilung vorlagen. ${ }^{41}$ Die nähere Betrachtung bezweckt, die relevanten Parallelen zum Papst-Vergleich und allfällige unterschiedliche Schlussfolgerungen herauszuarbeiten. Spezifische strafrechtstechnische Besonderheiten werden dabei weggelassen, soweit sie für das Aufsatzthema nicht von Belang sind.42 Die Fallkonstellationen waren die folgenden:

1) Ein anerkannter Kriegsdienstverweigerer zeigte sich über ein grosses Natomanöver in Mittelfranken bestürzt und schrieb auf ein Betttuch mit roter Farbe den Text: «A SOLDIER IS A MURDER».43

Das Transparent befestigte er an einer Strassenkreuzung am Ortsrand.

2) Ein anerkannter Kriegsdienstverweigerer verteilte in der Berufsschule seines Wohnorts, wo die Bundeswehr gerade eine Ausstellung durchführte, bebilderte Flugblätter mit folgendem Text: Sind Soldaten potentielle Mörder? Eines steht fest: Soldaten werden zu Mördern ausgebildet.

3) Ein Leserbriefschreiber schrieb: Ich erkläre mich in vollem Umfang mit

41 Urteil des BVerfG, 1 BvR 1476/91 (Fn. 36). Das Soldaten-sind-Mörder-Urteil von 1994 ist publiziert in: EuGRZ 1994, S. 463-465. Bei diesem Urteil ging es um einen ähnlichen Sachverhalt wie 1995: Ein anerkannter Kriegsdienstverweigerer hatte während des Golf-Krieges im Jahr 1991 auf der Rückseite seines Autos einen Aufkleber mit der Aufschrift «Soldaten sind Mörder» angebracht. Das «t» in dem Wort «Soldaten» war als Kreuz stilisiert. Unter dem Satz befand sich die faksimilierte Unterschrift «Kurt Tucholsky».

42 Beispielsweise die Besonderheiten der in der deutschen Lehre entwickelten Rechtsfigur von sogenannter Schmähkritik.

43 Auf die Besonderheit, ob eigentlich «Murderer» gemeint war, wird im Urteil eingegangen, Urteil des BVerfG, 1 BvR 1476/91 (Fn. 36), Rn. 7 ff. 
Herrn A. solidarisch und erkläre hiermit öffentlich: «Alle Soldaten sind potentielle Mörder!»

4) Vor einem Informationsstand der Bundeswehr hielten zwei Personen ein $1 \mathrm{~m} \times 3 \mathrm{~m}$ grosses Transparent hoch, auf dem stand: Soldaten sind potentielle MÖRDER.

Das untere Drittel des Wortes «Mörder» war mit dem Wort «Kriegsdienstverweigerer» unterlegt oder überschrieben.

\section{b) Erwägungen}

Wie bereits unter Ziff. 2 festgehalten, kann Kritik an gesellschaftlichen Verhaltensweisen im Rahmen der Meinungsäusserungsfreiheit auch in krasser Form vorgebracht werden. Wer gegen Abtreibung ist, darf dies unverblümt äussern. Auch eine überzogene oder gar ausfällige Kritik mache eine Äusserung für sich genommen noch nicht zur Schmähung, wird in «Soldaten-Mörder II» ausgeführt. Es müsse vielmehr hinzukommen, dass «bei der Äusserung nicht mehr die Auseinandersetzung in der Sache, sondern die Diffamierung der Person im Vordergrund» 44 stehe. Die Aussage müsse «jenseits auch polemischer und überspitzter Kritik in der persönlichen Herabsetzung bestehen».45 Dabei sei der objektive Sinn der Aussage zu ermitteln, und zwar nach dem «Verständnis eines unvoreingenommenen und verständigen Publikums».46 Das Gericht kam zum Ergebnis, dass die Gleichsetzung mit einem Mörder einen schwerwiegenden Angriff auf die Ehre und eine tiefe Kränkung ei-

44 Urteil des BVerfG, 1 BvR 1476/91 (Fn. 36), Rn. 122.

45 Urteil des BVerfG, 1 BvR 1476/91 (Fn. 36), Rn. 122.

46 Urteil des BVerfG, 1 BvR 1476/91 (Fn. 36), Rn. 125. nes Soldaten darstelle. Diese wiege besonders schwer, wenn die Bezeichnung im strafrechtlichen Sinn aber auch umgangssprachlich verwendet werde, «denn auch in diesem Fall bezeichnet er eine Person, die in einer sittlich nicht zu rechtfertigenden Weise zur Vernichtung menschlichen Lebens beiträgt oder bereit ist.» 47

26 Soweit jedoch eine verletzende Aussage eine weitere, gesellschaftskritische Aussageebene habe, müsse geprüft werden, ob diese Ebene im Vordergrund stehe, also überwiege. Bei den «Soldaten-sindMörder»-Urteilen musste sich das Gericht damit befassen, dass die Aussage «Soldaten sind Mörder» auf ein satirisches Zitat von Kurt Tucholsky48 zurückgeht, das auch als Kritik am Krieg bzw. der Pflicht der Soldaten gemeint ist und damit eine zweite Ebene des Pazifismus hat. Das Gericht folgerte: Aus dem Kontext und den aussertextlichen Umständen der konkreten Fälle ergebe sich, dass es beim Soldaten-Mörder-Vergleich «nicht um Kritik an einem besonders verwerflichen Individualverhalten oder gar an charakterlichen Mängeln von Soldaten» gehe, sondern um die problematischen Folgen von Soldatentum und Krieg.49 Aufgrund dieses überwiegenden gesellschaftskritischen Duktus der Äusserungen waren diese vom Bundesverfassungsgericht als zulässig bzw. als durch die Meinungsäusserungsfreiheit geschützt betrachtet worden.

47 Urteil des BVerfG, 1 BvR 1476/91 (Fn. 36), Rn. 129.

48 Publiziert in der Glosse «Der bewachte Kriegsschauplatz» (1931). Der verantwortliche Redaktor der Zeitschrift «Weltbühne», Carl von Ossietzky, wurde 1932 wegen «Beleidigung der Reichswehr» angeklagt, jedoch freigesprochen.

49 Urteil des BVerfG 1 BvR 1476/91 (Fn. 36), Rn. 130, $133,157$. 

beleidigungsfähig sei, kam das Bundesverfassungsgericht zum Ergebnis, dass die an eine Gruppe gerichtete verletzende Äusserung unter Umständen auch als «Angriff auf die persönliche Ehre der Mitglieder des Kollektivs» ${ }^{\circ}$ gesehen werden könne. Je grösser das Kollektiv sei, desto schwächer werde allerdings die persönliche Betroffenheit des einzelnen Mitglieds, «weil es bei den Vorwürfen an grosse Kollektive meist nicht um das individuelle Fehlverhalten oder individuelle Merkmale der Mitglieder» gehe. ${ }^{1} \mathrm{Im}$ Vordergrund könnte dann «die Kritik an sozialen Einrichtungen oder Phänomenen» stehen. ${ }^{2}$ Bei der Beleidigungsfähigkeit von Kollektiven stellt sich somit die Frage, wie sehr die persönliche Betroffenheit des einzelnen Mitglieds im Zentrum steht.

\section{Anwendung der Kriterien auf den Papst-Vergleich}

Folgenden stellt sich die Frage, ob die Argumentationslinie des Urteils auf das päpstliche Diktum übertragen werden kann. Es sind zwei Aspekte, die «Soldaten-Mörder II» für das vorliegende Thema interessant machen. Zum einen setzt sich das deutsche Bundesverfassungsgericht ausführlich mit der Stossrichtung bzw. Diffamierungsstärke der verletzenden Äusserungen auseinander. Bei der Stossrichtung oder bezweckten Wirkung der verletzenden Äusserung geht es darum, ob sie einzig auf Diffamierung abzielt, oder ob sie auch in einem anderen (zulässigen gesellschaftskritischen) Sinn $\mathrm{zu}$ verstehen ist. Zum anderen befasst sich das Gericht mit der Problematik,

5o Urteil des BVerfG, 1 BvR 1476/91 (Fn. 36), Rn. 134.

51 Urteil des BVerfG, 1 BvR 1476/91 (Fn. 36), Rn. 140.

52 Urteil des BVerfG, 1 BvR 1476/91 (Fn. 36), Rn. 140. dass die Adressaten der verletzenden Äusserungen ein Kollektiv darstellen. Damit hängt insbesondere die Frage zusammen, ob ein Kollektiv beleidigungsfähig ist.

29 Beide Aspekte spielen beim PapstVergleich von Abtreibung mit Auftragsmord eine Rolle: Einerseits die Frage, ob mit dem Vergleich die Verunglimpfung von betroffenen Frauen oder die Gesellschaftskritik des Vatikans an der Abtreibung überwiegt. Andererseits ob die betroffenen Frauen als Kollektiv beleidigungsfähig sind.

\section{a) Diffamierung von Frauen oder Gesellschaftskritik?}

30 Wenn der Papst Frauen, insbesondere auch solche, die gemäss ihrem nationalen Recht legal abtreiben, in einem Vergleich als Anheuernde von Auftragsmördern darstellt, so könnte darin eine Unterstellung von schwerstem kriminellem Verhalten gesehen werden. Denn wer einen Auftragsmörder engagiert, begeht strafrechtlich eine Anstiftung zu Mord, die grundsätzlich gleich zu bestrafen ist wie die Haupttat. Der Papst wirft den betroffenen Frauen dabei eine besonders abgefeimte Tat vor, indem sie sich die Hände nicht selber schmutzig machen, vielmehr einen Auftragsprofi anheuern, der den Mord gegen Entlohnung und ohne Skrupel ausführt.53

$5 3 \longdiv { \text { Papst Franziskus fällt damit noch hinter die diffe- } }$ renzierten Äusserungen von Papst Johannes Paul II. zurück. Dieser hatte in seinem «Brief von Johannes Paul II. an die Frauen» vom 29.6.1995 ausgeführt: «Wieviel Hochachtung verdienen angesichts solcher Entartungen hingegen die Frauen, die mit heroischer Liebe zu ihrem Kind eine Schwangerschaft austragen, die durch das Unrecht ihnen gewaltsam aufgezwungener sexueller Beziehungen zustande gekommen ist; was nicht nur im Rahmen der Greueltaten vorkommt, die 
31 Auch die heraufgesetzte Duldung von verletzender Kritik gegenüber Personen des öffentlichen Interesses greift hier nicht. Der Papst richtet seinen Vorwurf nicht etwa an eine Politikerin, die sich mit ihrer politischen Autorität für legale Abtreibung einsetzt.54 Vielmehr werden alle betroffenen Frauen durch den PapstVergleich angesprochen und verunglimpft. Der Vergleich erfolgt auch nicht in einer hitzigen politischen Diskussion, sondern anlässlich der Generalaudienz, in welcher nur der Papst Zugang zum Mikrophon hat.

Ob beim päpstlichen Vergleich eine zweite gesellschaftskritische Referenzebene überwiegt, in dem Sinne, dass der Papst die teilweise Legalisierung von Schwangerschaftsabbrüchen kritisiert und dazu aufruft, Frauen bzw. werdende Mütter so zu stärken, dass sie nicht zum Mittel der Abtreibung greifen müssen, ist zumindest fraglich. Für eine entsprechende Interpretation gibt es kaum Anhaltspunkte;

sich leider im Zusammenhang mit den auf der Welt noch immer so häufigen Kriegen ereignen, sondern auch in Situationen des Wohlstandes und des Friedens, die oft durch eine Kultur eines hedonistischen Permissivismus verdorben sind, in dem nur allzu leicht auch Tendenzen eines aggressiven Männertums gedeihen. Unter solchen Umständen ist die Entscheidung zur Abtreibung, die freilich immer eine schwere Sünde bleibt, eher ein Verbrechen, das dem Mann und der Mitwirkung des Umfeldes anzulasten ist, als eine den Frauen aufzuerlegende Schuld.»

54 Als Margrith Bigler-Eggenberger anfangs 1970erJahre als erste Richterin ans Bundesgericht gewählt werden sollte, titelte eine Zeitung aus der Ostschweiz, «Eine Mörderin ins Bundesgericht», weil sich Bigler-Eggenberger öffentlich für die Legalisierung der Abtreibung eingesetzt hatte (siehe dazu: Nina Fargahi, Margrith BiglerEggenberger: Mann schimpfte sie Mörderin, ZEIT Schweiz Nr. 36/2017, 31. August 2017). 20 Jahre später, 1993, wurde SP-Bundesratskandidatin Christiane Brunner angefeindet, indem behauptet wurde, sie sei an einer illegalen Abtreibung beteiligt gewesen. vielmehr sprechen gewisse Umstände klar dagegen: Aus dem Wortlaut der Papst-Rede ist zu erkennen, dass die Gleichstellung von strafloser Abtreibung, zu der schon von Gesetzes wegen 55 nicht leichtfertig 56 gegriffen wird, mit Auftragsmord nicht nur darauf abzielt, die teilweise Legalisierung von Abtreibungen zu kritisieren. Aufgrund der appellativen Botschaft, die in einen notorisch bekannten kirchenmoralischen Kontext eingebettet ist, kann der Vergleich nach objektiven Kriterien so verstanden werden, dass es vor allem auf den Einzelentscheid einer schwangeren Frau ankommt, niemals einen «Auftragsmörder zu engagieren». Der Fokus des Vorwurfs liegt somit schon auf dem inneren Entscheid einer Frau, eine (legale) Abtreibung vornehmen zu lassen.

33 Demgegenüber besteht beim SoldatenMörder-Vergleich eine andere Ausgangslage, indem der einzelne Soldat zunächst einmal nur einer allgemeinen Bürgerpflicht gehorcht, nämlich der Ableistung des ihm obliegenden Wehrdienstes. Er kann eine bewusste Entscheidung treffen und den Wehrdienst aus Gewissensgründen verweigern, so dass er nie im Krieg eingesetzt wird. Im Gegensatz dazu wird eine Frau nicht von alleine schwanger. Sie kann es unter Umständen nicht verhindern und steht dann vor der Situation, dass der Papst ihr, wenn sie eine Abtreibung vornehmen lässt, ein individuelles Fehlverhalten vorwirft, ohne ihr eine Lösung aufzuzeigen. 57

55 Vgl. Art. 118 und 119 StGB.

56 Z.B. Schwangerschaft nach Vergewaltigung, Gefährdung des Lebens der Mutter, drückende wirtschaftliche Not einer Familie.

57 Erwähnt sei hier, dass es keine Lösung, sondern eine von der Natur vorgesehene Folge der Schwangerschaft ist, das Kind zu gebären. Gerade 
$34 \mathrm{Ob}$ es beim päpstlichen Vergleich von Abtreibung mit Auftragsmord tatsächlich primär um den Schutz werdenden Lebens geht oder ob solche Aussagen auch Ausdruck der fehlenden Gleichstellung von Frauen und Männern in der römischkatholischen Amtskirche sind, ist eine weitere Frage, die je nach Antwort gegen das Vorliegen einer zweiten Referenzebene sprechen würde. Tendenziell ist die Gleichstellung der Frauen zumindest nicht prioritäres Thema der römischkatholischen Amtskirche.58 Der Heilige Stuhl hat seit 1970 Beobachterstatus im Europarat,59 hat die EMRK jedoch nicht unterzeichnet. Das von praktisch allen Nationen der Erde unterzeichnete Übereinkommen zur Beseitigung jeder Form

wenn das eigene Leben der betreffenden Frau in Gefahr ist, lösen auch das Gebären und eine Freigabe zur Adoption das Problem nicht.

58 Dies, trotz der berühmten Textstelle aus dem Brief des Apostels Paulus an die Gemeinde in Galatien: «Denn ihr alle, die ihr auf Christus getauft seid, habt Christus (als Gewand) angelegt. Es gibt nicht mehr Juden und Griechen, nicht Sklaven und Freie, nicht Mann und Frau; denn ihr alle seid ,einer in Christus Jesus.» Galater 3, $27 \mathrm{f}$. (Einheitsübersetzung). Darin wird auch auf theologischer Seite ein idealistischer Gleichstellungsansatz für die Gleichberechtigung der Geschlechter gesehen. Schüssler Fiorenza bezeichnet die Stelle als «theologischen Schlüsseltext», vgl. Elisabeth Schüssler Fiorenza, Grenzen überschreiten: der theoretische Anspruch feministischer Theologie, Münster 2004, S. 167 ff. Vgl. zur Thematik auch: Denise Buser, Die unheilige Diskriminierung, Eine juristische Auslegeordnung für die Interessenabwägung zwischen Geschlechtergleichstellung und Religionsfreiheit beim $\mathrm{Zu}-$ gang zu religiösen Leitungsämtern, Münster 2014, S. 60; dies., Dürfen Religionsgemeinschaften Frauen beim Zugang zu religiösen Ämtern diskriminieren? Zur Anwendbarkeit des CEDAWÜbereinkommens bei diskriminierenden religiösen Praktiken in der Ämterbesetzung, in: Anne Kühler et al. (Hrsg.), Quae Caesaris Caesari, quae Dei Deo? Bezüge von Recht und Religion im Wandel, Festschrift für Felix Hafner, Zürich/St. Gallen 2018, S. $61 \mathrm{ff}$.

59 Der Heilige Stuhl als Beobachterstaat. von Diskriminierung der Frau (CEDAWÜbereinkommen 60 ) hat der Vatikan ebenfalls nicht unterzeichnet.

35 Ein weiterer Umstand akzentuiert noch die Vehemenz der vergleichenden Bewertung von Abtreibung und Auftragsmord durch das Oberhaupt einer Weltkirche: Beim Papst (in seiner Funktion ${ }^{61}$ ) handelt es sich um eine universal anerkannte Autorität in Fragen der Moral, deren Verlautbarungen eine eminente Tragweite zukommt. Nicht ein Satiriker formuliert eine zugespitzte Aussage, die von unbekannten Zeitgenossen (Kriegsdienstverweigerern oder Leserbriefschreibenden) zitiert wird, sondern der Vertreter einer universalen Institution mit zweitausendjähriger Tradition stellt Frauen, die eine legale Abtreibung vornehmen, in die Nähe von Tätern und Täterinnen, die einen Auftragsmörder anheuern. Seine Äusserungen können von Abtreibungsgegnern als Aufforderung verstanden werden, Beschädigungen an Abtreibungskliniken vorzunehmen, Ärzte zu bedrohen, betroffene Frauen $\mathrm{zu}$ diffamieren. ${ }^{62}$ Sie wiegen deswegen besonders schwer.

$6 0 \longdiv { \text { UNO-Übereinkommen von 1979, das } 1 9 9 7 \text { in der } }$ Schweiz in Kraft trat (Wortlaut auf Deutsch in der Systematischen Rechtssammlung, SR 0.108).

61 Hier wird nicht einem Täterstrafrecht das Wort geredet, sondern es geht um den Papst in seinem Amt als Stellvertreter Christi in der Welt; nicht um seine persönliche Meinung als Privatperson.

62 So hält auch Urteil des EGMR 3687/10 vom 20.9.2018 in (Annen v. Deutschland [Nr. 3, siehe Fn. 20]) in $\S 31$ (letzter Satz) fest, dass Werturteile zu Hass und Übergriffen führen können. Zu erwähnen ist hier auch, dass in katholischen Ländern zuweilen, auch wenn nationales Recht unter gewissen Voraussetzungen einen Abbruch zulässt, nicht garantiert ist, dass Betroffene $\mathrm{Zu}$ gang zu den notwendigen ärztlichen Leistungen haben. Siehe dazu etwa das Urteil des EGMR 25579/o5 vom 16.12.2010 (A, B and C v. Ireland). Zuweilen werden sie wie im „Fall Agata“ gemobbt: Urteil des EGMR 57375/08 vom 30.10.2012 (P. und S. v. Polen). Siehe dazu Gab- 

Diktum zumindest fragen, ob mit der Formulierung «einen Auftragsmörder engagieren» nicht die Grenze von «scharfe[r] Kritik zur polemischen Diffamierung» 63 überschritten wird, und dadurch die Äusserung zumindest die Ränder einer Ehrverletzung berührt.

\section{b) Strafbare Kollektivbeleidigung?}

37 Bei der Aussage des Papstes ist zu beachten, dass sie nicht an eine, sondern an viele Einzelpersonen gerichtet war und somit die Frage einer Kollektivbeleidigung im Raum steht. ${ }^{64}$ Indem der Papst mit den beiden rhetorischen Fragen - die von Stimmen aus dem Publikum beantwortet wurden - die Zuhörerschaft bewusst einbezogen hatte, machte er klar, dass seine Äusserungen alle Menschen angehen, nicht nur einzelne, besonders hervorgehobene. Betroffen sind primär Frauen. ${ }^{65}$ Es liegt somit eine sehr grosse Gruppe vor, deren Beleidigungsfähigkeit aufgrund der Rechtsprechung nur mit $\mathrm{Zu}-$ rückhaltung angenommen werden kann. ${ }^{66}$ Doch auch hier legen Redestil, Autorität

riele Lesser, Abtreibungsverbot in Polen, taz 31.10.2012. Es ist deswegen relevant, wie sich der Papst positioniert.

63 Urteil des BVerfG, 1 BvR 1476/91 (Fn. 36), Rn. 159.

64 Mit Kollektivbeleidigung ist die Beleidigung mehrerer Einzelpersonen, nicht die Beleidigung einer Kollektiveinheit gemeint. Siehe dazu etwa Nikolaos Androulakis, Die Sammelbeleidigung, Darmstadt 1970, S. 9, der in diesem Zusammenhang den Begriff Sammelbeleidigung benutzt; Riklin (Fn. 29), vor Art. $173 \mathrm{~N} 52$.

65 Siehe 1. Einleitung.

66 Für die Schweiz siehe etwa BGE 124 IV 262, E. 2.a; Trechsel/Lieber (Fn. 29), vor Art. 173 $\mathrm{N}$ 12a; Riklin (Fn. 29), vor Art. $173 \mathrm{~N}_{52}$ je m.w.Nw. Für Deutschland siehe etwa Urteil des BVerfG, 1 BvR 1476/91 (Fn. 36), Rn. 139. Im französischen und angelsächsischen Recht wird Sammelbeleidigung auch nur mit grosser $\mathrm{Zu}-$ rückhaltung bejaht. Siehe dazu Androulakis (Fn. 64), S. 82 ff. mit rechtsvergleichenden Erläuterungen. des Papstes und die Einzelfallsituation einer Frau im Kontext einer Abtreibung nahe, dass gerade auch individuelles Fehlverhalten gemeint ist, und sich somit jede Frau bewusst sein muss, dass eine Abtreibung, die sie an sich vornehmen lässt, der Anheuerung eines Auftragsmörders gleichkommt. Die Brüskierung durch den Vergleich von Abtreibung und Auftragsmord ist für die einzelne Frau augenscheinlich vehement.67 Diese diffamierende Provokation kommt insbesondere durch das unausgesprochene, aber für ein allgemeines Publikum problemlos erkennbare Mitgemeintsein der individuellen Fehlleistung, zustande. Demgegenüber dürfte das Mitgemeintsein eines einzelnen Soldaten gegenüber einem TucholskyZitat, das von mehr oder weniger unbekannten Pazifisten und Pazifistinnen auf Flugblättern oder Transparenten veröffentlicht wird, wohl kaum so stark sein. Auch in diesem Punkt rückt der päpstliche Vergleich zumindest in eine problematische Nähe zur Ehrverletzung, auch wenn er alle betroffenen Frauen einbezieht.

38 Bei der Beurteilung der Strafbarkeit der Kollektivbeleidigung ist aber auch zu beachten, dass Ehrverletzungsdelikte im Wesentlichen die Austragung privater Fehden zum Inhalt haben. ${ }^{68}$ Geschützt wird die individuelle Ehre und nicht etwa wie bei der Rassendiskriminierung der öffentliche Friede.69 Bei der Güterabwä-

$67 \overline{\text { In der Schweiz hat der päpstliche Vergleich dazu }}$ geführt, dass sechs profilierte Theologinnen und Politikerinnen aus der Kirche ausgetreten sind (siehe Neue Zürcher Zeitung vom 20.11.2018).

68 Androulakis (Fn. 64), S. 88.

69 Entsprechend liegt keine Ehrverletzung vor, wenn behauptet wird, bestimmte Politiker hätten das Laufenthal verkauft, die Laufenthaler verseggelt und das Laufenthal dürfe nicht weiter von solchen Vasallen vertreten werden (BGE 116 IV 146). Ebensowenig ist die Bezeichnung «militante 
gung zwischen Meinungsäusserungsfreiheit und dem strafrechtlichen Schutz der Ehre ist deshalb zu unterscheiden, ob es um eine private Auseinandersetzung geht, dann ist die Ehrverletzung höher zu gewichten, oder ob es um die Bildung der öffentlichen Meinung geht, dann überwiegt die Meinungsfreiheit.70 Bei einer Kollektivbeleidigung kommt es deswegen darauf an, dass es eine Beziehung zwischen dem Täter und den Opfern gibt, die nahe legt, dass es um eine persönliche Konfrontation geht. Dies kann jeweils nur bei einem überschaubaren Personenkreis der Fall sein. ${ }^{11}$ «Wird [...] ein weiteres, ,unüberschaubares` Kollektiv angegriffen, so nähert sich die Äusserung demjenigen Bereich, der die ,Öffentlichkeit wesentlich berührt', und kommt folglich mit demjenigen Aspekt der Meinungsfreiheit in Berührung, der unantastbar ist: mit dem Recht, an dem ständigen Meinungskampf, der ,das Lebenselement der freiheitlichen demokratischen Grundordnung ist, teilzunehmen.»72 Entsprechend bejaht auch das schweizerische Bundesgericht die Strafbarkeit bei einer Kollektivbeleidigung nur, wenn erkennbar einzelne Personen betroffen sind. 73

39 Die Aussage des Papstes war nicht an individuell bestimmte Personen, sondern an einen unüberschaubaren Personenkreis gerichtet. Es geht damit nicht um eine persönliche Auseinandersetzung zwi-

Gruppe», die an der Sache vorbei politisiere, die Behörden übermässig beschäftige und den Steuerzahler sehr viel Geld koste, ehrverletzend (Urteil des Bundesgerichts 1C_438/2014 vom 19.3.2015, E. 3.5).

70 Androulakis (Fn. 64), S. 87 mit Verweis auf Urteil des Bundesverfassungsgerichts, 7, 212.

71 Androulakis (Fn. 64), S. 104.

72 Androulakis (Fn. 64), S. 105 f.

73 Das Bundesgericht hat beispielsweise die Strafbarkeit einer Ehrverletzung, die 73 Nationalräte betraf, bejaht (BGE 80 IV 166). schen dem Papst und einzelnen Personen, sondern im Kern um den Meinungskampf zwischen Pro-Life- und Pro-ChoiceBewegungen. Seine Äusserung ist deswegen nach dem Gesagten nicht als strafrechtliche Ehrverletzung zu qualifizieren.

\section{c) Fazit}

40 Die Äusserungen des Papstes wiegen insbesondere aus zwei Gründen schwer: Erstens wirft er Frauen, die eine (straflose) Abtreibung vornehmen lassen direkt ein strafbares (Anstiftung zum Mord), moralisch verwerfliches und feiges Handeln vor, und zweitens nimmt der Papst weder als Politiker (auch wenn er ein Staatsoberhaupt ist) an einer politischen Diskussion teil, noch vertritt er seine rein persönliche Meinung. Vielmehr äussert er sich als Oberhaupt einer Weltkirche und kann für sich kraft seines Amtes in Anspruch nehmen, besser als andere Menschen zu wissen, was aus einer christlichen Perspektive heraus richtig und was falsch ist. Der Papst-Vergleich könnte deswegen die Ränder einer Ehrverletzung berühren. Allerdings schützen die strafrechtlichen Ehrverletzungsdelikte die individuelle Ehre. Ehrverletzungen, die gegen ganze Gruppen gerichtet sind, sind deswegen nur strafbar, wenn erkennbar einzelne Personen betroffen sind.

\section{Sollte Geschlechterdiskriminie- rung strafbar sein?}

41 Von der hier untersuchten Aussage des Papstes ist eine grosse Zahl von Personen betroffen. Nicht alle, die sich beleidigt fühlen, sind aber auch in ihrer strafrechtlich geschützten Ehre verletzt.74 Dieser Befund

74 Androulakis (Fn. 64), S. 104. 
kann unbequem sein. Der Ehrverletzung ähnliche Angriffe auf Gruppen bleiben aber nicht grundsätzlich straflos. Sind Angehörige einer Rasse, Ethnie oder Religion betroffen, kommt eine Strafbarkeit wegen Rassendiskriminierung (Art. 261 ${ }^{\text {bis }}$ StGB) in Frage. Geschützt wird damit der öffentliche Friede. Die Strafnorm wird künftig möglicherweise auf die Diskriminierung wegen der sexuellen Orientierung ausgeweitet.75 Es stellt sich die Frage, ob er auch auf Geschlechterdiskriminierung bzw. generell auf Diskriminierung verschiedener Gruppen ausgedehnt werden sollte. Prima vista spricht gegen eine weitere Ausdehnung, dass Strafrecht generell als ultima ratio eingesetzt werden sollte. Zudem wären die Grenzen zur Meinungsäusserungsfreiheit noch schwieriger zu ziehen als bei der Kollektivbeleidigung, da als Rechtsgut nicht mehr «nur» die individuelle Ehre, sondern der öffentliche Friede geschützt wäre. Für eine Ausdehnung spräche, dass auch im Meinungskampf nicht alles erlaubt sein soll, und dem Meinungskampf ohnehin mehr geholfen ist, wenn er mit sachlichen Argumenten und nicht durch Diffamierung von Meinungsgegnern ausgetragen wird. Die Aussage des Papstes bietet aber zumindest Anlass, über einen generelleren Diskriminierungstatbestand nachzudenken.

\section{Fazit}

Der Papst-Vergleich von Abtreibung und Auftragsmord ist eine «schockierende Aussage ${ }^{76}$, sie erfüllt jedoch die (stren-

75 Gegen die vorgeschlagene Änderung, die auf die parlamentarische Initiative 13.407 zurückgeht, wurde innerhalb der Frist ein Referendum eingereicht.

76 Siehe dazu die Medienmitteilung vom 19. November 2018 von prominenten Katholikinnen, die auch aufgrund des Papst-Vergleichs aus gen) Tatbestandsvoraussetzungen der Ehrverletzungsdelikte nach schweizerischem Recht nicht. Denn geschützt wird dort die individuelle Ehre in der persönlichen Auseinandersetzung. Eine Kollektivbeleidigung ist deswegen nicht strafbar, solange keine Beziehung zwischen dem Täter und den Opfern besteht, die nahe legt, dass es um eine persönliche Konfrontation geht.

43 Ein Vergleich mit den deutschen «Soldaten-sind-Mörder»-Urteilen zeigt hingegen auf, dass bei der Aussage des Papstes zumindest eine problematische Nähe zu einer strafrechtlich relevanten Diffamierung vorliegt. Denn während beim Tucholsky-Zitat «Soldaten sind Mörder» die (pazifistische) Kritik an Kriegsführung und dem damit verbundenen obligatorischen Militärdienst im Vordergrund stehen, wird beim Papst-Vergleich gerade auch das angeblich individuelle Fehlverhalten der einzelnen Frau angeprangert.

44 Bei der Frage, ob der bisherige Rassismusstraftatbestand von Art. 261 $1^{\text {bis }}$ StGB um das Kriterium der Geschlechterdiffamierung $\mathrm{zu}$ erweitern wäre, ist $\mathrm{zu}$ beachten, dass bei diesem Strafdelikt zusätzlich noch eine Gefährdung des öffentlichen Friedens vorliegen muss. Dies stellt ein eigenes Thema dar und

der katholischen Kirche ausgetreten sind. Der Papst-Vergleich spiegle «die Grundhaltung der römisch-katholischen Amtskirche: Abtreibung ist in jedem Fall eine schwere Sünde, die mit Exkommunikation bestraft werden kann.» In der Medienmitteilung heisst es weiter, über Abtreibung könne man geteilter Meinung sein, empörend sei jedoch, Frauen in einer Notlage zu Kriminellen zu stempeln, während gleichzeitig Verhütungsmittel streng verboten seien. Werde eine Frau dann ungewollt schwanger, sei der Schutz des «ungeborenen Lebens» sakrosankt, die Lebenssituation der betroffenen Frauen werde dagegen komplett ausgeblendet. 
sprengt den Rahmen des vorliegenden Aufsatzes. $\mathrm{Zu}$ erwähnen ist immerhin, dass der öffentliche Friede auch durch die global vorhandene Problematik der massiven Gewalt an Frauen tangiert sein kann. Dabei besteht ein Zusammenhang zwischen Gewalt an Frauen und dem Absprechen von Selbstbestimmung in Fragen der weiblichen Körperlichkeit, wie es beim Abtreibungsthema zum Ausdruck kommt. Die Schweiz ist davon nicht ausgenommen, zumal Gewalt gegen Frauen und häusliche Gewalt auch hierzulande «ein gravierendes Problem [darstellen]: Täglich werden 11 Personen, 9 davon Frauen und Mädchen, in ihrer sexuellen Integrität geschädigt.»77 Das einschlägige statistische Material ist ebenfalls aufschlussreich.78 Insoweit wäre eine Ausweitung von Art. 261 ${ }^{\text {bis }}$ StGB zumindest prüfenswert.

77 Medienmitteilung des Eidgenössischen Büros für die Gleichstellung von Frau und Mann vom 13.11.2018. So erscheint es nur folgerichtig, dass die Schweiz am 1. April 2018 das Übereinkommen des Europarats zur Verhütung und Bekämpfung von Gewalt gegen Frauen und häuslicher Gewalt (die sogenannte Istanbul-Konvention, SR o.311.35) übernommen hat.

78 Häusliche Gewalt: Opfer von versuchten und vollendeten Tötungsdelikten in den Jahren 20092016 waren: 367 Frauen (61,3\%), 159 Männer (26,5\%) und 73 Kinder (12,2\%); Opfer von vollendeten Tötungsdelikten in den Jahren 20092016 waren: 124 Frauen (61,7\%), 42 Männer $(20,9 \%)$ und 35 Kinder (17,4\%); Angaben gemäss Eidgenössisches Büro für die Gleichstellung von Frau und Mann. 